\title{
Design Task Modeling and Task Allocation Method Research Based Multi- granularity Space in Distributed Network Environment
}

\author{
XiaoBo $\mathrm{CaO}^{1,2}$ \\ 1. Key Laboratory of Dynamics and Control of Flight \\ Vehicle, Ministry of Education, Beijing \\ 2. School of Aerospace Engineering, Beijing Institute of \\ Technology \\ Beijing, China \\ caoxiaobo198610@163.com
}

\begin{abstract}
To realize reasonable design task modeling and high efficiency task collaboration and allocation in complicated design process, a design task model based granular space is proposed, and cross-stage collaboration mode and task allocation method are discussed. Firstly, combining study results of Collaboration design and Cloud Manufacturing (CMfg), four dimension granular space of design task is defined and a general design task model is built. Secondly, cross-stage collaboration mode based task in distributed network environment is proposed. And then, design task allocation methods on both cross-stage synergy and single stage synergy are introduced. Finally, application prototype system is illustrated. Application results show that deign task model and task allocation in the multi-task collaboration mode are feasible.
\end{abstract}

Keywords-Granular Space;Design Task Modeling;Virtual Design Unit (VDU); Cross-stage Collaboration;Task Allocation

\section{INTRODUCTION}

Collaborative design and resources sharing in distributed network have become a tendency of complex product design. Design task modeling and task allocation are two key points in design process. Cloud Computing (CC) and Cloud Manufacturing (CM) [1] make it easily to realize design resources sharing and design task collaboration. There are a lot of researches oriented to complicated product design task modeling and multi-entity collaboration mode [2]-[5] at home and board. Most academics place different emphasis on resources selection and task scheduling. But several items are neglected as follows:

a) Multi-entity collaboration modes in complex product design or manufacturing are variable and uncertain. And typical modes are not summarized and classified.

b) Design task in design process is not modeled form the angle of multi-granularity.Task decomposition and combination are not reasonable.

c) Design resources organization and sharing. In the air vehicle design process, design resources discovery, selection, organization and scheduling go throughout all stages of design. There are not reasonable design resources organization unit and sharing method.

\author{
ChengDong $\mathrm{Xu}^{1,+2}$, ChunSheng $\mathrm{Hu}^{2}$ \\ 1. Key Laboratory of Dynamics and Control of Flight \\ Vehicle, Ministry of Education, Beijing \\ 2. School of Aerospace Engineering, Beijing Institute of \\ Technology \\ Beijing, China \\ xucd@bit.edu.cn \\ jeadean@163.com
}

Our research aims to develop a general design task model based granular space in distributed network environment. Cross-stage task synergy mode and task allocation method are also studied in the study results of our team, and it makes efficient to finish complicate design jobs in such a way as to minimize the design cost. Remainder sections of this paper are organized as follows. Section II introduces key issues of design task modeling and multi-task collaboration mode, and our emphasis are stated in this section. Section III introduces the granular space definition of design task, and granular space model of design task is also displayed. Section IV firstly put forward the design task model based granular space. Key elements of design task model are also introduced. And then multi-stage collaboration mode based task in distributed environment is illustrated and discussed. Section $\mathrm{V}$ discussed task allocation methods on both crossstage synergy and within-stage synergy. Finally, demonstration instances and application result are displayed. Future works are also concluded.

\section{DESIGN TASK GRANULARITY SPACE}

In the macroscopic design process of complicated product, design tasks refer to different stages, different activity and different function object. To realize reasonable modeling of design task and efficient task scheduling for cross-design-stage, four dimension task granularity space is developed to describe design stage granularity information, object structure granularity information, function granularity information and Cost\&Time\&Quality (CTQ) granularity information. As shown in Figure 1, design task granular space model with four aspects of information into the $4 \mathrm{~d}$ coordinate is introduced with the following definitions.

$$
\mathrm{Gran}_{\mathrm{x}}=(\mathrm{G} 1, \mathrm{G} 2, \mathrm{G} 3, \mathrm{G} 4)
$$

Design stage granularity dimension (G1) : it describes the classification of the design stages according to the product development sequence. Each value in G1 coordinate denotes one stage in design process. Design stages granularity types include product project demonstration, conceptual design, specific design and prototype test and finalize design.

Object structure granularity dimension (G2) : it describes structure granularity size of design task object 
according to the structure complicate degree. Each value in G2 coordinate denotes task object structure granularity from simple to complicate, which include element of part, part, component and product.

Function granularity dimension (G3) : it describes the function granularity which design task can realize according to design activity destination. Each value in G3 coordinate denotes one stage in design process.

CTQ granularity dimension (G4) : it describes the CTQ granularity when design task is executed according to the product development sequence. Each value in G4 coordinate denotes design task quality level.

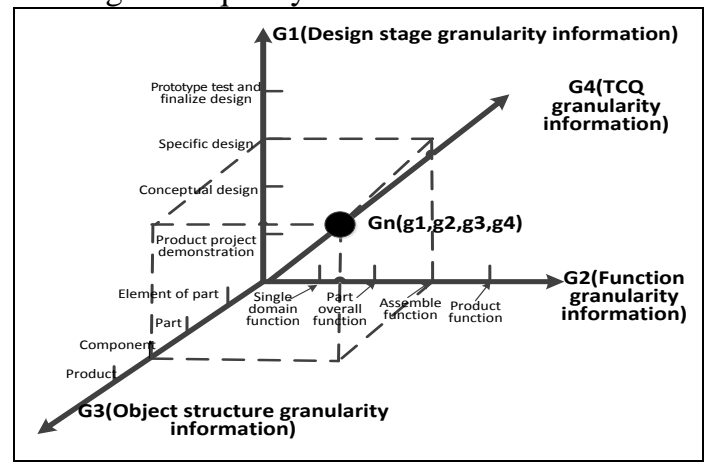

Figure 1. Design task granular space model diagram

\section{DESIGN TASK MODELING AND MUlti-STAGE COLLABORATION MODE REASERACH}

\section{A. Design Tasks Modeling Based Granular Space}

According to granularity space and design task attribution, design task is built as show as Figure 2.

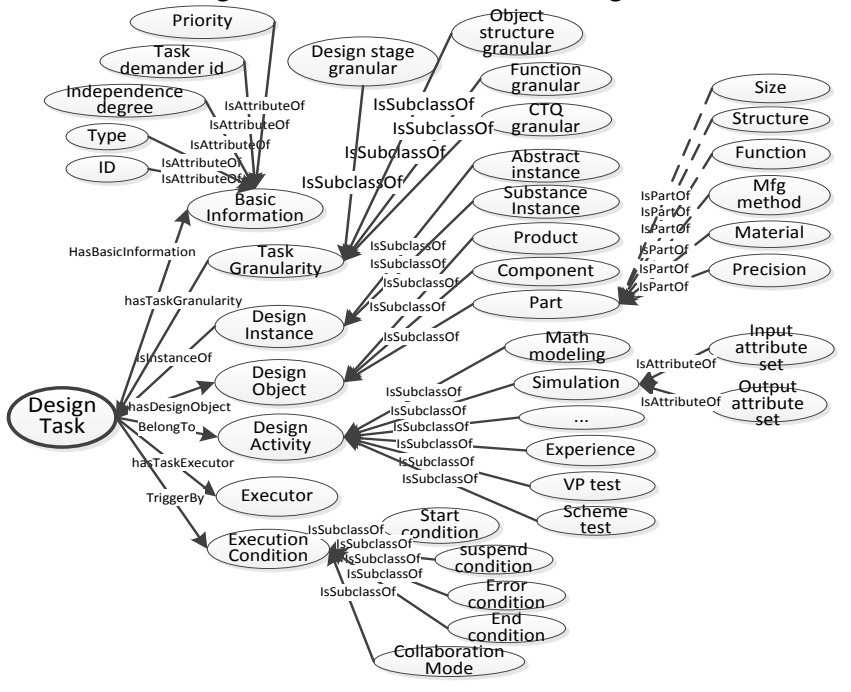

Figure 2. Design task model

a) Design task model structure

Refer to papers [6]-[9], design task model based multigranular space(DTMMGS) is composed of basic information, design instance, design object, design activity, executor, execution conditions and collaboration mode. $D T$ is used to denote design task model, and it is expressed with 7-tuple as follow.

$$
D T=(B, G, I, O, A, E, C)
$$

Among them, $\mathrm{B}$ denotes basic design information including task ID, task name, independence degree, priority, task demander id.

$G$ denotes granularity information of design task. $G=(g l$, g2, g3, g4). gl=(StageType, PhaseLevel) denotes stage granular dimension information, $g 2=($ Object - Type, Structure level) denotes design object structure granular dimension information, g3=(Function Type, FunctionLevel) denote function granular dimension information, $g 4=($ TCQlevel, Time, Cost, Quality) denotes Time\&Cost\&Quality granular dimension information.

$I=($ AbsInstanceId, SubInstanceId $)$ denotes the design task instances including abstract instance and substance instance.

$O=($ ObjID, ObjName, ObjClass, Size, Structure, Function, Mat,Mfgmethod) denotes object information of design task, it includes object Id, object name, object category, object size, object structure, function, material and Mfg method.

$A=($ ActId, Name, Type, Method, InputSetId, OutputSetId $)$ denotes design activity information of design task, it includes activity id, name, type, method, activity input attribute set id and activity output attribute set id.

$E=($ ExecutorId,Name, Structurelevel,Function, Affiliation, DesignAbility) denotes design task executor information, it includes executor id, name, structure level, function, affiliation and design ability.

$C=($ Execuation, Transfer, Collaboration, Start, Suspend, Er rorDeal,End) denotes condition and constrain of design task in different status. It includes task execution condition, transfer condition, collaboration condition, start condition, suspend condition, error dealing condition and end condition.

b) Design task executor

To realize efficient use of design resources, Virtual Design Unit (VDU)[10][11] is taken as the basic task execution unit. According to design activity types, VDUs are divided into design scheme demonstration, geometric modeling, grid generating, programming, mathematic modeling, engineering analysis, simulation, experiment, test, scheme check and prototyping. Single design resources are the elements of VDU. According to design task requirement, VDUs are dynamically generated by organizing different design resources. VDUs are connected by design task sequence executed in design process. Design tasks are assigned with short time, low cost and equilibrium load.

\section{B. Multi-stage Design Collaboration Based Task in Distributed Network Environment}

Complicate product design businesses have becoming more and more complex with high expensive cost and resources investment. As for the most product enterprises and institutes, limited financial capital, techniques, manpower, design resources severely restrict the development and business. Resources sharing and multi-task cooperation under the distributed network environment enable enterprises or institutes to focus more on core design businesses without considering design resource scales. This paper proposes a multi-stage collaboration mode based 
design task as shown in Fig 3. The mode has three major characteristics:

a) Cross-stage synergy \& synergy within single stage. It achieves Cross-stage synergy from the angle of design stage granularity and time sequence. And it realizes synergy within stage from the angle of object structure granularity and function granularity.

b) Aggregation and sharing of design resources. Distributed network environment[10] build a virtual design resources pool to gathering different kinds of virtual design resources. Design resources are organized in the form of VDU with certain agglomeration design ability. VDUs are allocated and shared to finished complicated synergy tasks with low cost and high efficiency.

c) Dynamic Combination and allocation of design tasks. Design tasks are monitored synchronously and design tasks in the same granularity level can be dynamic combined to execute by following minmiun cost principle.

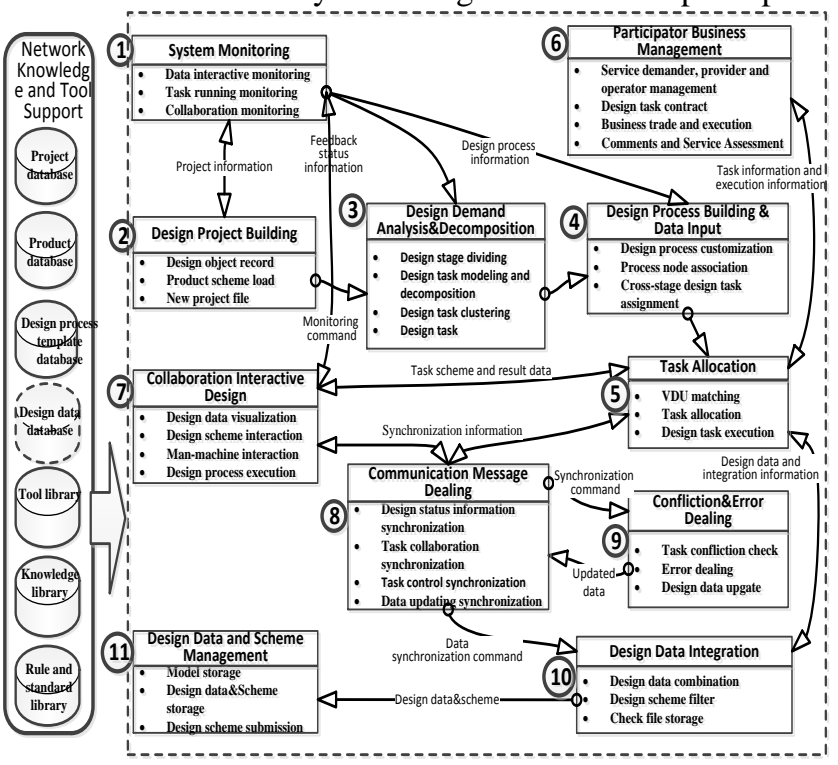

Figure 3. Multi-stage collaboration mode based design task in distributed network environment

\section{TASK SCHEDULING METHOD RESEARCH}

Task allocation in distributed network environment is to determine the executor for each design task. It is the same meaning with task assignment. The purpose of task allocation is to finish design tasks with low cost and short time. When some similar design tasks are accepted in design platform, the platform chooses the candidate executors from design resource pool. In the paper, VDU is taken as the basic execution unit of design tasks. Both cross-stage task allocation and single stage task allocation are considered in the paper as follow.

\section{A. Multi-granular Design Tasks Allocation In Cross-stage}

From the angle of design stage granularity dimension and TCQ granularity dimension, product design process is composed of project preparatory stage, conceptual design stage, specific design stage, test and finalized design stage in Figure 4. Design stage indicates and optimization target are denoted with $S C i\left(t_{i}, c_{i}, q_{i}\right) . t_{i}$ denotes time parameter set index, $c_{i}$ denotes cost parameter set index, $q_{i}$ denotes quality parameter set index. Minimizing of three component indicators weight sum $\operatorname{Min}\left(\alpha_{1} \cdot \sum\left(t_{i}\right)+\alpha_{2} \cdot \sum\left(c_{i}\right)+\alpha_{3} \cdot \sum\left(q_{i}\right)\right)$ is taken as overall design target.

From the angle of object structure granularity dimension, structure granularity scales of design object are divided into specific domain level, part level, component level and product overall level from fine to rough.

From the angle of function granularity dimension, function granular scales of design task are divided into specific domain function level, part overall function level, assembling function level and production function level.

In the same stage, design task is allocated from coarse scale to fine scale. Design task execution sequence and time are dispatched by task Stage optimization indicators. Design task interaction relation is determined by design sequence. Among different design stage, design schemes are delivered in the form of XML, CAD file and HTML.

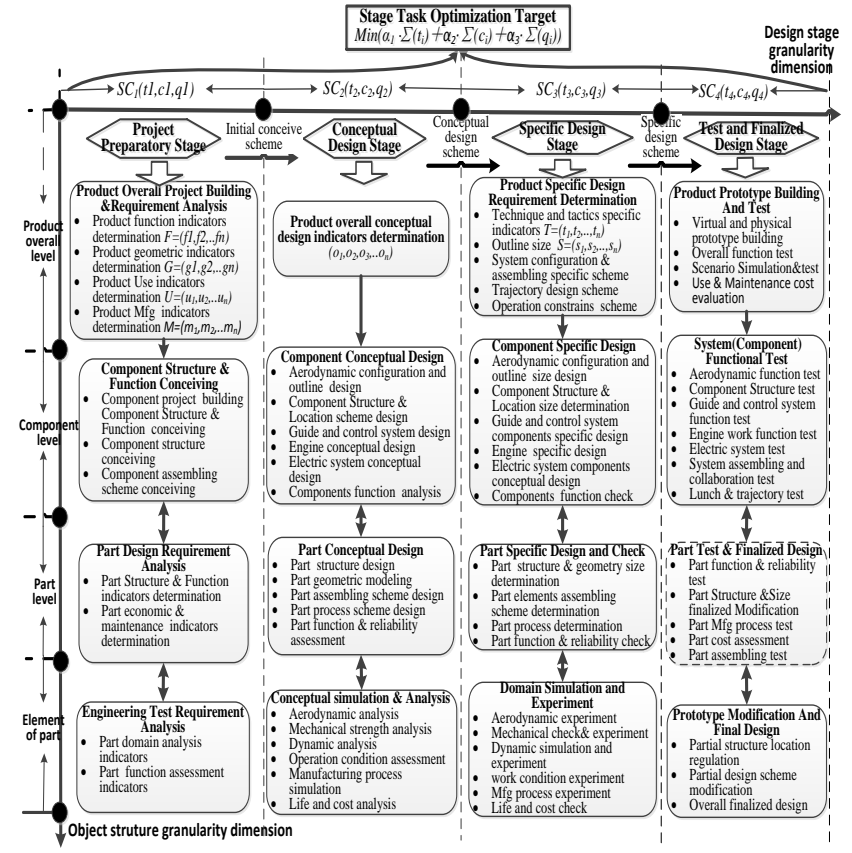

Figure 4. Design task flow cross different stages in granular dimensions

\section{B. Design Tasks Allocation in Single Design Stage}

As for similar tasks allocation in single design stage, the problem of assigning design tasks to VDUs in such a way as to maximize the overall performance is a challenging one[11]. There are three main phases of design task scheduling process. Phase one is tasks decomposition and classification. In the phase, product design tasks or part design tasks are divided into orderly subtasks set. Phase two is VDU discovery and VDU task allocation. VDU discovery provides a list of available VDUs, and VDU task allocation involves selection of feasible VDUs and mapping of tasks to 
VDUs. The third phase includes task execution and task control. In the second phase, the ultimate goal of tasks scheduling is to make sure that proper tasks number of different design task types are executed by the VDUs at the right time and low cost[12].

The value of relevance degree between task and VDU decides whether one VDU is suitable for executor the task. In general, there exists correlation between tasks and VDU. Let $\mathrm{T}$ be the correlation matrix of task and VDU, the element $\mathrm{T}_{\mathrm{ij}}$ denotes the correlation factor between the $i$ th task and $j$ th VDU. $P=\left(p_{1}, p_{2}, \ldots, p i\right)$ denotes the attributes set of task, and $Q=\left(q_{1}, q_{2}, \ldots, q_{j}\right)$ denotes ability attribute set of VDU.

\section{1) Task Scheduling Problem Description}

On condition that VDU task allocation problem in a single design stage is described under the following assumption.

a) Optimization objective for VDU task allocation problem is to finish certain number of design tasks with some VDUs at the lowest total cost. And it must meet the constraints of delivery cycle and work load equilibrium.

b) VDU can carry out one process or more processes. Each task only can be finished by one VDU.

c) Number of design task is more than number of $V D U$.Different types of design task is relative independent.

VDU task allocation is the most link of realizing low cost collaboration design. Mathematical formula of design task scheduling problem is stated as follow. Formula (1) denotes overall cost of VDUs to finish all similar design tasks. Overall cost is the sum of financial cost and executed time with certain weight factor.

$$
\begin{aligned}
& \operatorname{minf}=\delta \square \sum_{r} \sum_{i}\left[e_{r i}^{\square} x_{r i} \square\left(C_{\mathrm{ri}}+C_{\mathrm{ri} \rightarrow i+1}\right)\right] \\
& +(1-\delta) \square \sum_{r} \sum_{\mathrm{i}}\left(e_{r i}^{\square} \square\left(x_{r i} \square T_{\mathrm{ri}}+T_{\mathrm{ri} \rightarrow i+1}\right)\right) \\
& \text { s. t. } \quad \sum_{i} e_{r i} \square x_{r i}=N_{i}, N_{i} \geq 0 \\
& \sum_{r} \sum_{\mathrm{i}}\left(x_{r i} T_{\mathrm{ri}}+T_{\mathrm{ri} \rightarrow i+1}\right) \leq \mathrm{D}_{\text {off }}, \forall r, i \\
& \frac{\sum_{r} \sum_{\mathrm{i}}\left(x_{r i} T_{\mathrm{ri}}+T_{\mathrm{ri} \rightarrow i+1}\right)}{D_{\text {off }}} \leq L_{\mathrm{r}}
\end{aligned}
$$

Among them, $r=(1,2, \cdots, N+)$ denotes the number of VDU. $i=(1,2, \cdots, N+)$ denotes number of design task type. $\mathrm{Ni}=(\mathrm{N1}, \mathrm{N} 2, \ldots, \mathrm{Ni})$ denotes the number of $i$ th type of design task. $e_{r i}$ denotes matching degree value of rth VDU and ith design task. $C_{r i}$ denotes single execution cost when $r$ th VDU carry out $i$ th type design task. $\mathrm{Cr} i \rightarrow i+1$ denotes the single prepare cost when $r$ th VDU transfer from $i$ th type design task to $i+1$ th type design task. $T_{r i}$ denotes execution hour number when $r$ th VDU carry out $i$ th type design task. Tri $\rightarrow$ $i+1$ denotes the prepare hour number when $r$ th VDU transfer from $i$ th type design task to $i+1$ th type design task.
$D_{\text {off }}$ denotes design time of all design task. $L_{r}$ denotes max work hour load per day of $r$ th VDU. $\delta$ is the weight factor of financial cost and design time.

2) VDU Allocation Method Statement

According to above optimization formula, VDU allocation method combined ACO[13]-[15] and GA[16]-[18] is described as follow:

a) Task set is created after task decomposition.On condition that multiple VDU dealing with multiple kinds of tasks,different design tasks are classified and agglomerated. Purpose of task allocation is to select reasonable number of every kind of task for every available VDU.

b) A VDU set is selected according to the requirements of design task type and design characteristic. In VDU scheduling, some means of estimating a task's execution cost and money must be used. Cost, execution time and shipping time of each working procedure are identified, when VDU carry out some design activities. Refer to paper[18], cost calculation formula of VDU is showed as follows:

$C_{\mathrm{ri}}=\frac{\sum_{k=1}^{k}\left(n_{e k} \square c_{e k}\right)+\sum_{s=1}^{s}\left(n_{m s} \square c_{m s}\right)+\sum_{t=1}^{t}\left(n_{t t} \square c_{t t}\right)+c_{a u x}}{L_{\mathrm{ri}}}$

$c_{r i}$ denotes the design activity cost when $\boldsymbol{r}$ th VDU carry out the $i$ th task. $n_{e k}$ and $c_{e k}$ denote the number and hour cost of the $k$ th kind of equipment. $n_{m s}$ and $c_{m s}$ denote the number and hour cost of the sth kind of man. $n_{t t}$ and $c_{t t}$ denote the number and hour cost of the $t$ th kind of auxiliary tools. $c_{a u x}$ denotes the sum of other affix cost. $L_{r i}$ denotes the hourly workload of $r$ th VDU when $r$ thVDU take in ith design task.

c) Solution variable initialization. Candidate VDU could not execute all kind of task,So relevance correlation matrix isintroduced as formula(7).If $r$ th VDU is suitable for $i$ th task, $e_{r i}=0$; else $e_{r i}=1 . x_{r i}$ denotes the number that $r$ th VDU carry out $i$ th type design task. $x_{r i}$ is taken as the solution variable. And then, initial value of $x_{r i}$ is selected randomly from the value range $\left[0, N_{i}\right]$.Meanwhile $x_{r i}$ value must meet the formula(8). All the $x_{r i}$ construt the design task number matrix of task allocation optimization as formula(9).

$$
\begin{gathered}
E=\left[\begin{array}{cccc}
e_{11} & e_{12} & \ldots & e_{1 i} \\
\ldots & \ldots & \ldots & \ldots \\
e_{r i} & e_{r i} & \ldots & e_{r i}
\end{array}\right] \\
x_{r i}=N_{i}-\sum_{r=1}^{r-1} x_{r i}\left[e_{r i}\right. \\
X=\left[\begin{array}{cccc}
x_{11} & x_{12} & \ldots & x_{1 i} \\
\ldots & \ldots & \ldots & \ldots \\
x_{r 1} & x_{r 2} & \ldots & x_{r i}
\end{array}\right]
\end{gathered}
$$

d) According to ACO,optimization method is executed. $m$ is assumed as the number of ant. $n$ denotes the number of ant iteration. Every design task type is task as the city node for ant visiting. When $m$ th ant visit the $i$ th type of design 
tasks, ant allocate task number of all design task types to executed by all VDUs.Every ant start randomly from one of $i$ design types. And then, ant select visit sequence and design task number schem until all design task types are executed. All design tasks must be arranged when ant finish the whole journey. When ant completely visit all task types, design task nummber matrix in the path is generated as one solution. Minimum delivery time is used to judge whether one solution meet the charge time. If formula(4) is not satisfied, it demonstrates that current task number schemes fail to meet the deliver time limitation. Under such situation, deliver time limitation or outsourcing processing is supposed to adjusted and executed.

e) Next city selection of mth ant in t time. When mth ant moves from jth type of design task to kth type of design task in time.Taboo table is built to exclude task types that ant has visited. The line between $j$ th types and $k$ th types is denoted with $(j, k)$,and pheromone is denoted with $\tau_{j k \text {. Initial }}$ value of $\tau_{j k}$ is set as $\tau_{j k}(0)=1$. Motion transition probability that ant select the jth design types is formulated as formula(10).

$$
P_{j k}^{m}(\mathrm{t})=\left\{\begin{array}{l}
\frac{\left(\tau_{j k}\right)^{\alpha} \square\left(\eta_{j k}\right)^{\beta}}{\sum_{l \notin T a b u}\left[\tau_{j l}(t) \square\left(\eta_{j l}\right)^{\beta}\right]} \\
0
\end{array}\right.
$$

f) Local pheromone updating. When ant moves from jth type of design task to kth type of design task, it leaves pheromone in the line $(j, k)$.The more ants move from line $(j, k)$, the more pheromone leave. When one ant move from line $(j, k)$, local pheromone is updated by the formula(11)(12).

$$
\begin{aligned}
& \Delta \tau_{j k}^{m}=\left\{\begin{array}{l}
\frac{Q}{d_{\text {all }}}, \text { when ant } \mathrm{m} \text { vist } \mathrm{jk} \text { node } ; \\
0, \quad \text { else }
\end{array}\right. \\
& \tau_{j k}(t+1)=\rho \cdot \tau_{j k}(t)+\Delta \tau_{j k}^{m}
\end{aligned}
$$

When ant visits from start design task type to the current jth design task type, $j$ - 1 lines are visted. The total cost $C_{j}$ and total time $T_{j}$ of $j-1$ lines are calculated by path calculation formula(12) and formula(13).Walking path length of ant $d_{\text {all }}$ is calculated by weighted function formula(15). To consider effect from both financial cost and time, weight factor $\delta$ is introduce to calculate the comprehensive overall cost of the path.

$$
\begin{aligned}
C_{j} & =\sum_{j=1}^{\mathrm{j}}\left(e_{j i}\left[\sum_{i=1} x_{j i} \square\left(C_{\mathrm{ji}}+C_{\mathrm{ji} \rightarrow i+1}\right)\right)\right. \\
T_{j} & =\sum_{j=1}^{j} \sum_{i=1}^{i}\left(e_{j i} \square\left(x_{j i} \square T_{\mathrm{ji}}+T_{\mathrm{ji} \rightarrow i+1}\right)\right) \\
d_{\text {all }} & =\delta \cdot C_{j}+(1-\delta) \cdot T_{j},(\delta \in[0,1])
\end{aligned}
$$

g) The adaptive crossover and mutation operation. In order to avoid the local optimal, crossover and mutation operation[17] have to be carried out to the components of solution after pheromone updating to achieve the global optimal.

Every ant finish completely path visiting, a design task number matrix is generated and it is taken as one solution.According to path length formula(15), two solution are selected to carry out crossover by certain probability. Suppose that $m$ th ant path length is denoted by $d_{\text {over, } m}$ and its probability of being chosen is formulated as formula(16). In the traversal paths of $m$ ants, the smaller the path length ant travel, the choose probability is smaller. Smaller choose probability means better solution.

$$
\mathrm{P}_{m}=d_{\text {over }, m} / \sum_{d=1}^{m} d_{\text {over }, d}
$$

In the crossover operation, supposed $x(1)=\left(x_{11}, x_{21}, \ldots\right.$, $\left.x_{r 1}\right)^{T}$ and $x(2)=\left(x_{12}, x_{22}, \ldots, x_{r 2}\right)^{T}$ are 1 th and 2 th column elements set of selected solution $(\boldsymbol{X})$.Crossover is executed between column elements set $x(1)$ and $x(2)$. Real crossover probability is calculated by formula(17)-(19).

$$
\begin{aligned}
& \mathrm{P}_{\text {cross }}=P_{\text {sysem }} \square p_{c} \\
& \mathrm{P}_{c}=d_{i} / \sum_{h=1}^{m} d_{h} \\
& d_{i}=\sum_{r=0}^{r}\left[e_{r i} \square x_{r i} \square\left(C_{\mathrm{ri}}+C_{\mathrm{ri} \rightarrow i+1}\right)\right]
\end{aligned}
$$

$p_{\text {system }}$ is generated by system. Comparison probability $p$ $\in[0,1]$ is also randomly generated. If $p>p_{\text {system, }}$ crossover operation is executed. $\alpha \in[0,1]$ is generated randomly. $x(1)$ and $x_{r}(2)^{\prime}$ are used to replace $x(1)$ and $x(2), x(1)^{\prime}=\alpha \cdot x(1)_{+}(1-$ $\alpha) \cdot x(2) ; x(1)^{\prime}=\alpha \cdot x(2)_{+}(1-\alpha) \cdot x(1)$.

After executed crossover operation, mutation operation is randomly executed between two elements of column elements set by certain system probability. Real mutation probability is calculated by formula(20).

$$
\mathrm{P}_{\text {mutation }}=P_{M} \square p_{m}
$$

$P_{M}$ is generated by system, and Comparison probability $p$ $\in[0,1]$ is also randomly generated. If $p>p_{M}$, mutation operation is executed. $\varepsilon \in[0,1]$ is generated randomly, $x_{11}=\varepsilon \cdot x_{11+}(1-\varepsilon) \cdot x_{12} ; x_{12}{ }^{\prime} \varepsilon \cdot x_{12+}(1-\varepsilon) \cdot x_{11}$.

h) Optimization and iteration.After abover crossover and mutation,two new solutions are generated, and walking path length is calculated. Design task number matrix of the shortest walking path in $m$ solutions is selected as the initial solution of next iteration.

i) Global pheromone updating. For the shortest line of path, pheromone is updated by formula $(21)(22)$.

$$
\begin{aligned}
\tau_{j k}^{\text {new }} & =\alpha \square \tau_{j k}^{\text {old }}+\Delta \tau_{j k}^{m} \\
\Delta \tau_{j k}^{m} & =\left\{\begin{array}{l}
\frac{Q}{d_{\text {shortest }}} \\
0, \quad \text { else }
\end{array}\right.
\end{aligned}
$$

j) Finishing Iteration and outputting task allocation scheme. 


\section{CONCLUSION}

Some interfaces of vehicle design platform based on DTMMGS and collaboration mode is illustrated in Figure 5.

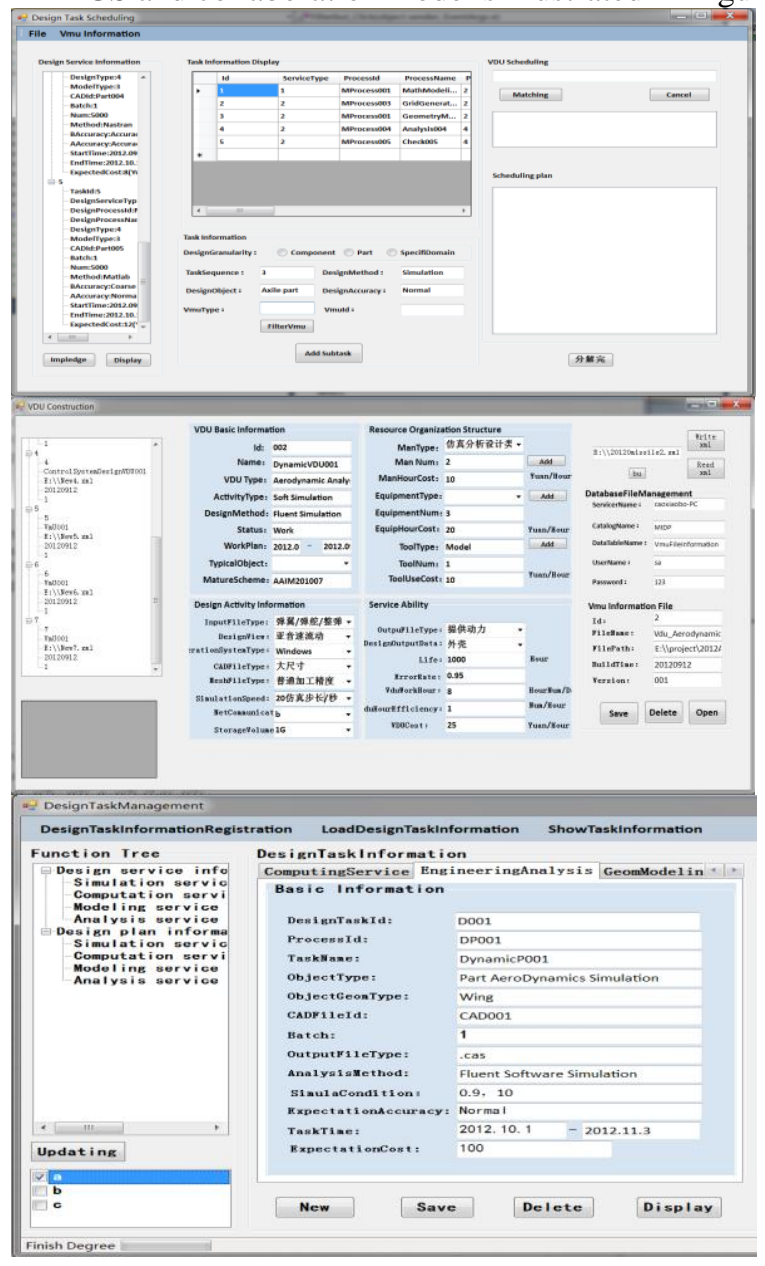

Figure 5. Part interfaces of Prototype system

Design task model based multi-granular spcace and task allocation method in both cross-stage and single stage have been applied to air vehicle integrated design platform based on VDU. VDU allocation methode combined ACO and GA is applied to design task scheduling module of prototype system, and application result is acceptible.

Future research include: (1)Design process optimization method. (2) Design service ability refine model.

\section{ACKNOWLEDGMENT}

This work is supported by the National High-Tech.R\&D Program, China (No.2011AA120505) and the National Natu ral Science Foundation, China (No.61173077).

\section{REFERENCES}

[1] Bohu Li, Lin Zhang, Shilong Wang, et al, "Cloud manufacturing: a new service-oriented networked manufacturing model," Computer Integrated Manufacturing Systems, CIMS, Vol.16, 2010, pp.1-7
[2] Changfeng Yao, Dinghua Zhang, Kun Bu, "Collaborative manufacturing task information model and its model building method for complex parts," Computer Integrated Manufacturing Systems, CIMS, 2009,16(8):1649

[3] Chih-Hsuan Wang, Jiun-Nan Chen, "Using quality function deployment for collaborative product design and optimal selection of module mix," Computers \& Industrial Engineering,Vol.63, Dec.2012,pp.1030-1037,doi:10.1016/j.cie.2012.06.014

[4] Lijun Song, Yu Yang, Jie Yang, "Research on Task Dynamic Scheduling for Product Collaborative Design Based on Multi-rule," Journal of Chongqing University,Vol.31,Jan.2008,PP.2-4

[5] Maud Rio, Tatiana Reyes, Lionel Roucoules, "Toward proactive (eco) design process: modeling information transformations among designers activities," Journal of Cleaner Production,Vol.39,Jan.2013, pp. 105-116 ,doi: 10.1016/j.jclepro.2012.07.061

[6] Anning Zhou, Yi Liu, "The Method of Design Process Modeling for Airplane Based on the Cooperative Pattern," Mechanical Science and Technology for Aerospace Engineering, Vol.31,2012,pp.323-329

[7] Yonghui Guo, "Collaborative Innovation and Collaborative Mode for Design Chain," 2011 Fourth International Symposium on Computational Intelligence and Design, 2011:137-140, doi: 10.1109/ISCID.2011.136

[8] Chunsheng $\mathrm{Hu}$, Chengdong $\mathrm{Xu}$, Xiaobo Cao, et al, "Study of classification and modeling of virtual resources in Cloud Manufacturing," Applied Mechanics and Materials, Frontiers of Manufacturing and Design Science II, 2012, pp.2274-2280.

[9] Chunsheng $\mathrm{Hu}$, Chengdong Xu, Pengfei Zhang, " Study On Simulation Model Virtualization in Cloud Computing Environment," J. HuaZhong Univ. of Sci. \& Tech.(Natural Science Edition),Vol.40,Dec.2012,pp.135-140

[10] Xiaobo Cao, Chengdong $\mathrm{Xu}$, Chunsheng $\mathrm{Hu}$, "Virtual manufacturing unit in cloud Manufacturing," Computer Integrated Manufacturing Systems, CIMS, Vol.18, July.2012, pp.1415-1425

[11] Xiaobo Cao, Chengdong Xu, Chunsheng Hu, "Study on Architecture of Missile Design Service Platform Based on Virtual Design Unit," Advanced Materials Research, Vol.655-657,2013,pp.20572064,doi:10.4028/www.scientific.net/AMR.655-657.2057

[12] A.Y.Abdelaziz, Reham A.Osama, S.M.El-Khodary, Bijaya Ketan Panigrahi, "Distribution Systems Reconfiguration Using the HyperCube Ant Colony Optimization Algorithm," Computer Science.2011(7077):257-266.

[13] PengYeng Yin, JingYu Wang, "Ant colony optimization for the nonlinear resource allocation problem," Applied Mathematical and Computation,Vol.174,Mar.2006,pp.14381453,doi:10.1016/j.amc.200 5.05 .042

[14] S.K.Chaharsooghi, Amir H.Meim, Kerman, "An effective ant colony optimization algorithm(ACO) for multi-objective resource allocation problem(MORAP)," Applied Mathematics and Computation,Vol.200, Jun.2008,pp.167-177,doi: 10.1016/j.amc.2007.09.070

[15] Jiyong Ding, Zhuofu Wang, “Applying Ant Colony Optimization to Multiple Resources Leveling Problem,'ESEP2011.Sept.2011, Singapore,pp.2045-2053,doi: 10.1016/j.egypro.2011.11.385

[16] YingKai Wang, XiaoDong An, "Logistics modeling and simulation based on hereditary algorithm," Journal of North University of China. 2005(26):421-424.

[17] Rahul Putha, Luca Quadrifoglio, Emily Zechman, "Comparing Ant Colony Optimization and Genetic Algorithm Approaches for Solving Traffic Signal Coordination under Oversaturation Conditions," Computer-Aided Civil and Infrastructure Engineering,Vol.27, Jan.2012, pp.14-28, doi:10.1111/j.1467-8667.2010.00715.x

[18] B. Chandra Mohan, R. Baskaran, "A survey:Ant Colony Optimization based recent research and implementation on several engineering domain," Expert Systems with Applications, 2012 (39) :4618-4627 